\title{
Strategies of reducing input sample volume for extracting circulating cell-free nuclear DNA and mitochondrial DNA in plasma
}

\author{
Weijie Chen, Fengfeng Cai, Bei Zhang \\ and Xiao Yan Zhong* \\ Laboratory for Gynecological Oncology, Department of \\ Biomedicine, University Women's Hospital, University of \\ Basel, Basel, Switzerland
}

\begin{abstract}
Background: Circulating cell-free (ccf) DNA in blood has been suggested as a potential biomarker in many conditions regarding early diagnosis and prognosis. However, misdiagnosis can result due to the limited DNA resources in Biobank's plasma samples or insufficient DNA targets from a predominant DNA background in genetic tests. This study explored several strategies for an efficient DNA extraction to increase DNA amount from limited plasma input.

Methods: Ccf plasma DNA was extracted with three different methods, a phenol-chloroform-isoamylalcohol (PCI) method, a High Pure PCR Template Preparation Kit method and a method used for single cell PCR in this group. Subsequently, the total DNA was measured by Nanodrop and the genome equivalents (GE) of the GAPDH housekeeping gene and MTATP 8 gene were measured using a multiplex real-time quantitative PCR for the quantitative assessment of nDNA and mtDNA.

Results: Instead of 400-800 $\mu \mathrm{L}$ (routine input in the laboratory), $50 \mu$ Lof plasma input enabled the extraction of ccf DNA sufficient for quantitative analysis. Using the PCI method and the kit method, both nDNA and mtDNA could be successfully detected in plasma samples, but nDNA extracted using protocol for single cell PCR was not detectable in $25 \%$ of plasma samples. In comparison to the other two methods, the PCI method showed lower DNA purity, but higher concentrations and more GE of nDNA and mtDNA.

Conclusions: The PCI method was more efficient than the other two methods in the extraction of ccf DNA in plasma. Limited plasma is available for ccf DNA analysis.
\end{abstract}

Keywords: circulating cell-free DNA; mitochondrial DNA; nuclear DNA.

\footnotetext{
*Corresponding author: Xiao Yan Zhong, Laboratory for Gynecological Oncology, Department of Biomedicine, University Women's Hospital, University of Basel, Hebelstr, 20, 4031 Basel, Switzerland Phone: +41 612659 248, Fax: +41 612659 399, E-mail: xzhong@uhbs.ch Received July 7, 2011; accepted October 3, 2011; previously published online October 31, 2011
}

\section{Introduction}

Circulating cell-free (ccf) DNA in blood has been suggested as a potential biomarker for many conditions $(1,2)$. Quantitative and qualitative assessments of ccf DNA in plasma are gaining increasing importance as tools for bloodbased diagnosis and monitoring varieties of diseases (3-5). The quantitative changes, such as nuclear DNA (nDNA) and mitochondrial DNA (mtDNA) and qualitative changes, such as tumor-specific methylated DNA, viral DNA, microsatellite alterations and oncogene mutations were observed in plasma and serums of various conditions. Ccf DNA assessments offer a high diagnostic specificity in cancer, pathological pregnancies, inflammatory diseases, trauma and transplant graft rejection (6-11). This might become a valuable prognostic marker providing the possibility of examining diseases from genetic materials in the circulation. Thus, the quantitative and qualitative assessments of ccf DNA are important tools for diagnosing and tracking the diseases and their progressions.

However, there were many problems faced in extracting ccf DNA from plasma. Firstly, there are no commercially available extraction kits intended for ccf DNA and especially the special genes in plasma; therefore, the extraction kits for whole blood DNA were often used instead. Furthermore, the concentrations of ccf DNA reportedly vary from 6 to $650 \mathrm{ng} / \mathrm{mL}$ approximately in the plasma of healthy men $(12,13)$. The wide range and low concentrations suggest that the currently performed DNA extraction method is not ideal for analysis of ccf DNA. Since a standard method for extracting ccf DNA in the plasma does not exist, further studies and clinical applications of ccf DNA were limited.

Another problem encountered was the limited DNA resources and the minute amounts of DNA targets which hindered the detection of target genes at sufficient amounts. Since the levels of ccf DNA in plasma varies depending on diseases, the more quantitative and qualitative assessments of ccf DNA investigated the better the analysis of relating ccf DNA to specific diseases. Therefore, more DNA targets and more plasma were needed for genetic tests. However, valuable plasma or serum samples from Biobank are normally limited and orders are in quantities insufficient for DNA extraction. Moreover, this led to inaccuracies when conducting qualitative assessments of ccf DNA which can easily lead to misdiagnosis, especially when very low concentrations of DNA targets are observed in the case of tumor DNA, fetal DNA and oncogene mutations.

The aim of this study was to explore an efficient method of extracting ccf DNA for further analysis of ccf DNA. We compared the quantification of total DNA, nDNA and mtDNA 
extracted in plasma by three different methods: the column kit method, the phenol-chloroform-isoamylalcohol (PCI) method and the method used for single cell PCR successfully to amplify genes from single cell. The three methods were used to select an optimal ccf DNA extraction for reducing input sample volume while increasing the extracted DNA concentration necessary for ccf DNA analysis. The concentration of ccf DNA was measured by Nanodrop and the GE of the GAPDH housekeeping gene and MTATP 8 gene were measured using a multiplex real-time quantitative PCR for the quantification of nDNA and mtDNA, which was successfully developed in our laboratory (14).

\section{Materials and methods}

\section{Sample collection}

The plasma samples used in this study were obtained from the Biobank of Basel University Hospital. Blood samples were collected into tubes containing EDTA- $\mathrm{K}_{3}$ as anticoagulant and processed within $2 \mathrm{~h}$ after vein-puncture. Subsequently, they were processed immediately by centrifugation at $1600 \times \mathrm{g}$ for $10 \mathrm{~min}$. The plasma layer was transferred to a new Eppendorf tube and centrifuged again at maximum speed $(16,000 \times g)$ for $10 \mathrm{~min}$. Plasma samples were divided into aliquots of $400 \mu \mathrm{L}$ each and stored at $-80^{\circ} \mathrm{C}$. The study was approved by the Local Ethic Committees.

\section{DNA extraction}

The extractions of ccf DNA in 20 plasma samples were conducted using three protocols: the PCI method, High Pure PCR Template Preparation Kit (Roche) method and the method used for single cell PCR. The PCI method is an organic solvent extraction-based DNA preparation (15); $50 \mu \mathrm{L}$ plasma was diluted into $550 \mu \mathrm{L}$ water and an equal volume of PCI (25:24:1) was added. The mixture was centrifuged at top speed for 1-2 min to separate the phases. Subsequently, the aqueous phase was moved to a new tube with two volumes of ice-cold $100 \%$ ethanol to precipitate the DNA. After 1 min full speed centrifugation, the supernatant was removed and DNA dissolved in 12.5 $\mu \mathrm{L}$ tris-EDTA buffer. The cef DNA of the second aliquot was extracted using a High Pure PCR Template Preparation Kit, which was a silica-based column DNA preparation and performed according to the kit instrument. The extracted DNA from $200 \mu \mathrm{L}$ plasma was dissolved in $50 \mu \mathrm{L}$ tris-EDTA buffer. For the third aliquot, the protocol for single cell PCR was used (16). Ccf DNA was extracted, amplified and quantified in the same tube; $5 \mu \mathrm{L}$ plasma was added to a PCR tube containing $7.5 \mu \mathrm{L}$ of $400 \mathrm{ng} / \mu \mathrm{L}$ Proteinase $\mathrm{K}$ and $17 \mu \mathrm{mol} / \mathrm{L}$ sodium dodecyl sulfate. The solution was incubated at $50^{\circ} \mathrm{C}$ for $1 \mathrm{~h}$, followed by $99^{\circ} \mathrm{C}$ for 30 min to extract DNA, degrade protein and use for quantitative analysis.

\section{Total DNA quantitative determinations}

The concentrations of different DNA dilutions were detected by a Nanodrop 1000 spectrophotometer (Thermo Scientific) firstly. Because the composition and quality of ccf DNA were unidentified, the multiplex real-time PCR was conducted subsequently. The ccf DNA extracted by protocol for single cell PCR was mixed by proteins and amplified by real-time PCR directly; therefore, its concentration was not measured.

\section{Amplification and quantification of nDNA and $\mathrm{mtDNA}$ in plasma samples}

Real-time PCR assays are widely used for quantitative assessment of DNA in plasma or serum because it is shown to be simple and robust with a detection limit of about $1 \mathrm{ng} / \mathrm{mL}$ and high inter-assay reliability (17), but the spectrophotometric measurements were not suitable for such minute quantities of DNA in plasma (5). Therefore, the GE of nDNA and mtDNA were assessed with the multiplex real-time PCR. This process could perform two assays in a single tube at the same time and be an efficient and accurate method for quantifying the ccf nDNA and mtDNA (14).

A total of $5 \mu \mathrm{L}$ of DNA dilution was used as a template for the TaqMan real-time PCR analysis by an ABI PRISM 7000 Sequence Detection System (Applied Biosystems, ABI). The amount of nDNA was quantified using the following primer pairs and VIC-labeled TaqMan MGB-probe for the GAPDH housekeeping gene. The primers were 5-CCC CAC ACA CAT GCA CTT ACC-3 (forward primer) and 5-CCT AGT CCC AGG GCT TTG ATT-3 (reverse primer) and the probe was 5-(MGB) TAG GAA GGA CAG GCA AC (VIC)-3. For determining mtDNA, a sequence of the MTATP 8 gene starting at locus 8446 was amplified and quantified. The primers were 5-AAT ATT AAA CAC AAA CTA CCA CCT ACC-3 (forward primer) and 5-TGG TTC TCA GGG TTT GTT ATA-3 (reverse primer) and the probe was 5-(FAM) CCT CAC CAA AGC CCA TA (MGB)-3. The multiplex real-time PCR was performed in a total reaction volume of $25 \mu \mathrm{L}$, containing $5 \mu \mathrm{L}$ of DNA, $12.5 \mu \mathrm{L}$ of TaqMan Universal PCR Master Mix, four primers and two probes. Our standard TaqMan PCR conditions have been described in our previous publication (14), which involved a 2 min incubation at $50^{\circ} \mathrm{C}$, followed by an initial denaturation step at $95^{\circ} \mathrm{C}$ for $10 \mathrm{~min}$ and 40 cycles of $1 \mathrm{~min}$ at $60^{\circ} \mathrm{C}$ and $15 \mathrm{~s}$ at $95^{\circ} \mathrm{C}$.

A standard curve of a diluted DNA, one negative control and one calibrator DNA, were included in each run. The concentrations of ccf nDNA were estimated according to the standard curves and drawn using the known concentration of human genomic DNA. The concentrations of ccf nDNA were expressed as GE per $\mathrm{mL}$ of plasma (18). Relative quantities of ccf mtDNA could be estimated using fold change of $\mathrm{GE}_{\mathrm{nDNA}}$ and also expressed as $\mathrm{GE}$ per $\mathrm{mL}$ of plasma.

\section{Statistical analysis}

In all cases, experiments were replicated in triplicate and data was presented as median, range and fold change and analyzed with the SPSS software. The Wilcoxon, Mann-Whitney and Kruskal-Wallis tests were used to compare the amounts of ccf DNA in different groups categorized by PCI method, kit method and protocol for single cell PCR. A p-value below 0.05 was considered statistically significant.

\section{Results}

\section{Total DNA quantitative determinations}

The purity and concentration of DNA dilution extracted by two different protocols were different. The ratio of 260/280 of DNA dilution extracted by kit was higher than that by PCI method ( $1.65 \pm 0.15$ vs. $1.06 \pm 0.21)$. However, the concentration was less than that by PCI method $(23.91 \pm 8.82 \mathrm{ng} / \mathrm{mL}$ vs. $407.32 \pm 239.36 \mathrm{ng} / \mathrm{mL}$ ). Because the DNA extracted by protocol for single cell PCR was amplified directly, the concentration and purity of DNA were not investigated. 


\section{Amplification and quantification of $\mathrm{nDNA}$ and mtDNA in plasma samples}

Ccf DNA extracted by three different methods was amplified, quantified and subsequently compared for the differences in the quantification of nDNA and mtDNA. Using the PCI method and kit method for DNA extraction, both nDNA and mtDNA were successfully detected in plasma samples; mtDNA was also successfully detectable with the protocol for single cell PCR, while nDNA was not in $25 \%$ of plasma samples. The standard curves using a known concentration of human genomic DNA containing six concentration points for both mtDNA and nDNA are shown in Figure 1. The average slope of standard curve of mtDNA or nDNA was approximately -3.3 (nearly $100 \%$ efficiency) in multiplex real-time PCR.

The ccf DNA equivalents in the paired plasma samples were determined by multiplex real-time PCR, calculated according to the standard dilution curves, expressed as GE/mL, and shown in Table 1. The GE of nDNA and mtDNA extracted by PCI were greater than that by kit, respectively, $(9094.84 \mathrm{GE} / \mathrm{mL}$ vs. $4498.81 \mathrm{GE} / \mathrm{mL}, 119315.70 \mathrm{GE} / \mathrm{mL}$ vs. $21741.76 \mathrm{GE} / \mathrm{mL})$, in the plasma, although a lower volume of plasma was used $(50 \mu \mathrm{L}$ vs. $200 \mu \mathrm{L}$ ). The GE of mt DNA extracted by PCI method was approximately 5.5-fold and the GE of nDNA was approximately 2-fold of the kit method. By the protocol of single cell PCR, the mtDNA could be detectable in all samples (1647.06 GE/mL), with the nNDA in $75 \%$ of the samples $(525.45 \mathrm{GE} / \mathrm{mL})$. The examples of amplification curves of mtDNA extracted by three methods were shown in Supplementary Figure 1. The cycle threshold $(\mathrm{Ct})$ value of mtDNA extracted by the PCI method was less than that by the kit method.

\section{Discussion}

In this study, we aimed to explore an efficient method to extract the ccf DNA in limited plasma samples. Three methods for extracting DNA were involved. Although the PCI method

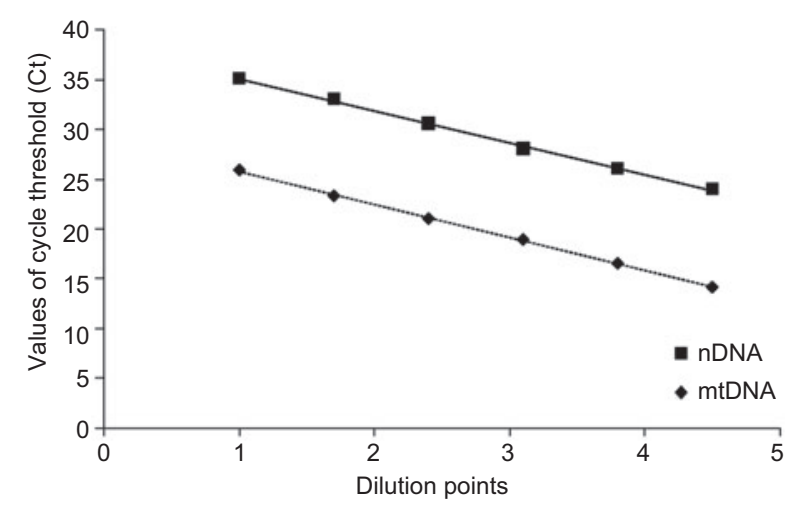

Figure 1 Multiplex real-time PCR kinetics for amplifying mtDNA and nDNA on serial dilutions.

The figure shows reproducible standard dilution curves for identification of the mtDNA and nDNA. The standard curves with average slopes was approximately -3.3 (nearly $100 \%$ efficiency) in multiplex real-time PCR. provided less purity, the concentration of ccf DNA measured by Nanodrop was more than that by the kit method. The 260/280 ratio of ccf DNA extracted by the PCI method was less than that by the kit method. This indicated that there might be contamination of residual ethanol, protein and others in the dilution extracted by PCI method. It might be easier to get contamination with the PCI method while removing the aqueous phase containing the DNA to a new tube. However, the kit could bind nucleic acids with column and remove contaminating components in a series of rapid "wash and spin" steps. Therefore, it had a better purity but might lose some unbound DNA. Considering the contamination in the DNA dilutions, the result that the concentration of ccf DNA extracted by PCI method was more than that by kit method might not prove convincing. Thus, the genome equivalents, the quantification of nDNA and mtDNA were conducted subsequently.

The results of quantifying nDNA and mtDNA showed that the GE of ccf mtDNA extracted by PCI method were greater in number and that the mtDNA: nDNA ratio determined of the PCI method group was also higher than that of the kit group, although there was not any statistical differences between the two methods when comparing their GE of nDNA. These results indicated that the column isolation by kit might reduce total ccf DNA in ways that wasted more mtDNA than nDNA. This could cause the under-estimation of the GE of mtDNA. Most column DNA extraction kits rely on high concentrations of chaotropic salts and low $\mathrm{pH}$ to extract DNA because the negatively-charged DNA phosphate backbones could bind to positively-charged silica gel beads. However, the salt concentrations, $\mathrm{pH}$, fat and wash buffer ethanol contents could influence DNA binding in a size-dependent manner $(15,19)$. The column kits were optimized for the isolation of DNA fragments by more than $50 \mathrm{~Kb}$ (20). Moreover, the RNAase contained in the column kits could minimize the quantity of mtDNA (15). Thus, the column kits seemed unsuitable for mtDNA quantification but did not affect nDNA quantification. This also might be the reason for the wide range of quantitative assessments discussed in the previous papers, i.e., the mean ccf DNA concentrations of $6-20 \mathrm{ng} / \mathrm{mL}$ or $650 \mathrm{ng} / \mathrm{mL}$ were measured in the plasma of healthy men by different methods (2).

The PCI method could provide a high ccf DNA capture efficiency, though more time-consuming and subject to variable technical prowess. It was less dependent on DNA size and enabled more accurate assessment of small size DNA, like mtDNA. Since ccf DNA consists of fractions of different sizes (21), using isolation methods that capture all of the DNA fractions is of the utmost importance for the reliability of all downstream experiments and their comparability (22). Therefore, the PCI method seems to be preferable in capturing and isolating of mtDNA. Although that insufficient phenol could cause oxidation and contaminants of residual ethanol, proteins and divalent cations might inhibit PCR, the amplification efficiency of nDNA and mtDNA were nearly $100 \%$ and the GE of ccf DNA extracted by PCI was still higher in the study. Moreover, the DNA extracted by the PCI method could be concentrated by decreasing the volume of dissolved buffer while the test kit method does not allow all of the DNA 
Table 1 Circulating cell-free mtDNA and nDNA extracted by three different methods.

\begin{tabular}{|c|c|c|c|c|c|c|}
\hline \multirow[t]{2}{*}{ Sample } & \multicolumn{3}{|l|}{ nDNA, GE/mL } & \multicolumn{3}{|l|}{ mtDNA, GE/mL } \\
\hline & PCI method & Kit method & Single cell PCR & PCI method & Kit method & Single cell PCR \\
\hline 1 & 4295.94 & $20,239.84$ & 525.45 & $30,759.80$ & $58,856.34$ & 5139.51 \\
\hline 2 & 7737.59 & 2562.57 & 166.69 & $59,792.12$ & 8800.53 & 3931.89 \\
\hline 3 & 2958.04 & 5140.07 & 75.70 & $20,177.02$ & $24,620.50$ & 362.60 \\
\hline 4 & $11,564.47$ & 1751.88 & 93.88 & $128,144.40$ & 7355.91 & 408.11 \\
\hline 5 & $18,569.92$ & 3857.55 & 1335.58 & $147,533.20$ & 725.82 & 1401.98 \\
\hline 6 & 8078.00 & 3110.42 & Undetectable & $119,759.70$ & 569.24 & 576.83 \\
\hline 7 & 3539.28 & 399.50 & Undetectable & $28,511.16$ & $11,129.20$ & 1779.62 \\
\hline 8 & $11,731.63$ & 1678.05 & 192.41 & $247,679.70$ & $39,309.04$ & 1550.01 \\
\hline 9 & 1268.43 & 154.94 & Undetectable & $65,938.06$ & 1579.80 & 139.31 \\
\hline 10 & $462,349.60$ & $24,566.92$ & 932.93 & $5724,129.00$ & $1259,508.00$ & $12,639.23$ \\
\hline 11 & 8931.70 & 3439.13 & 1252.05 & $173,517.20$ & $31,604.21$ & 7913.36 \\
\hline 12 & $43,932.05$ & $21,130.30$ & 3946.85 & $415,067.50$ & $18,912.15$ & $21,269.29$ \\
\hline 13 & $585,885.40$ & $294,195.10$ & 1364.64 & $2825,861.00$ & $352,292.40$ & $43,067.32$ \\
\hline 14 & $11,481.78$ & $29,184.01$ & 149.68 & $75,650.31$ & $30,213.19$ & 840.86 \\
\hline 15 & 9257.98 & $12,695.19$ & Undetectable & $29,460.24$ & 4744.35 & 261.96 \\
\hline 16 & 5724.21 & 1030.12 & 236.92 & $121,690.80$ & $21,153.37$ & 1744.12 \\
\hline 17 & $14,654.39$ & $11,399.68$ & 164.31 & $118,871.70$ & $22,330.16$ & 385.42 \\
\hline 18 & 3775.40 & 2454.58 & Undetectable & $15,526.15$ & 3471.30 & 313.44 \\
\hline 19 & 6283.90 & $25,464.36$ & 1279.30 & $70,603.09$ & $35,270.93$ & $17,093.18$ \\
\hline 20 & $380,914.00$ & $38,608.75$ & 4790.64 & $3026,262.00$ & $484,669.00$ & $335,511.70$ \\
\hline Median & 9094.84 & 4498.81 & $25.45^{\mathrm{a}}$ & $119,315.70$ & $21,741.76^{\mathrm{a}}$ & $1647.06^{\mathrm{a}}$ \\
\hline and range & $(525.45-4498.81)$ & (154.94-294,195.10) & $(75.70-4790.64)$ & $(15,526.15-5724,129.00)$ & $(569.24-1259,508.00)$ & $(139.31-335,511.70)$ \\
\hline
\end{tabular}

a Statistical significances between the method and PCI method $(\mathrm{p}<0.05)$; mtDNA, mitochondrial DNA; nDNA, nuclear DNA; PCI method, phenol-chloroform-isoamylalcohol method; kit method, High Pure PCR Template Preparation Kit (Roche) method.

binding to columns to be washed down with fewer volumes of dissolved buffers.

The single cell PCR was a very sensitive method, but little contamination could verify the results (16). By using only $5 \mu \mathrm{L}$ volume of plasma, the mtDNA was detectable, but the concentration of mtDNA was less than that of the PCI method. This indicated that the DNA in the action tube might be combined or broken down by the contaminants, because all the salts and proteins in the plasma were put in the detection system. Moreover, because the nDNA was less than mtDNA, it can be detectable only in $75 \%$ of samples. Also, the input volume of plasma was limited by the size of tube, therefore, the improvement of detection sensitivity of nDNA by increasing input of plasma might not be feasible.

Increased clinical importance of ccf DNA was found not only for the diagnosis, prediction and prognosis of varied diseases but also for monitoring patients in disease surveillance and during treatment. As result, efficient isolation procedures of ccf DNA and sensitive determination procedures were demanded. The PCI method demonstrated higher efficiency in extracting of ccf DNA and can be an important procedure for detecting the quantitative and qualitative change of ccf DNA in limited DNA resource and targets. The PCI method needed only $50 \mu \mathrm{L}$ plasma and could gain more concentration and genome equivalents of DNA dilution by column kit method which used $200 \mu \mathrm{L}$ plasma. Although the protocol for single cell PCR uses even less plasma, it was not as sensitive as the PCI method due to the inhibition of protein or salt. Thus, the PCI method might be considered a suitable approach for extracting ccf DNA in plasma.

\section{Acknowledgments}

This work was supported by China Scholarship Council. We are grateful to Matt Ji for proofreading the manuscript. We thank Vivian Kiefer, Corina Kohler, Zeinab Barekati and Hongbo Chen for their kind assistance.

\section{Conflict of interest statement}

Authors' conflict of interest disclosure: The authors stated that there are no conflicts of interest regarding the publication of this article. Research support played no role in the study design; in the collection, analysis and interpretation of data; in the writing of the report; or in the decision to submit the report for publication.

Research funding: None declared.

Employment or leadership: None declared.

Honorarium: None declared.

\section{References}

1. Sozzi G, Conte D, Leon M, Ciricione R, Roz L, Ratcliffe C, et al. Quantification of free circulating DNA as a diagnostic marker in lung cancer. J Clin Oncol 2003;21:3902-8.

2. Jung K, Fleischhacker M, Rabien A. Cell-free DNA in the blood as a solid tumor biomarker - a critical appraisal of the literature. Clin Chim Acta 2010;411:1611-24. 
3. Catarino R, Ferreira MM, Rodrigues H, Coelho A, Nogal A, Sousa A, et al. Quantification of free circulating tumor DNA as a diagnostic marker for breast cancer. DNA Cell Biol 2008;27:415-21.

4. Ye L, Ma GH, Chen L, Li M, Liu JL, Yang K, et al. Quantification of circulating cell-free DNA in the serum of patients with obstructive sleep apnea-hypopnea syndrome. Lung 2010;188:469-74.

5. Swarup V, Rajeswari MR. Circulating (cell-free) nucleic acids - a promising, non-invasive tool for early detection of several human diseases. FEBS Lett 2007;581:795-9.

6. Lo YM, Tein MS, Lau TK, Haines CJ, Leung TN, Poon PM, et al. Quantitative analysis of fetal DNA in maternal plasma and serum: implications for noninvasive prenatal diagnosis. Am J Hum Genet 1998;62:768-75.

7. Konorova IL, Veiko NN, Ershova ES, Antelava AL, Chechetkin AO. Haemodynamic role of blood-plasma circulating cell-free DNA and contained therein high-molecular-weight CpG-rich fraction in pathogenesis of arterial hypertension and atherosclerosis obliterans of carotid arteries. Angiol Sosud Khir 2009; 15:19-28.

8. Allen D, Butt A, Cahill D, Wheeler M, Popert R, Swaminathan R. Role of cell-free plasma DNA as a diagnostic marker for prostate cancer. Ann NY Acad Sci 2004;1022:76-80.

9. Lam NY, Rainer TH, Chiu RW, Joynt GM, Lo YM. Plasma mitochondrial DNA concentrations after trauma. Clin Chem 2004;50:213-6.

10. Zhong XY, von Muhlenen I, Li Y, Kang A, Gupta AK, Tyndall A, et al. Increased concentrations of antibody-bound circulatory cellfree DNA in rheumatoid arthritis. Clin Chem 2007;53:1609-14.

11. Lo YM, Tein MS, Pang CC, Yeung CK, Tong KL, Hjelm NM. Presence of donor-specific DNA in plasma of kidney and livertransplant recipients. Lancet 1998;351:1329-30.

12. Altimari A, Grigioni AD, Benedettini E, Gabusi E, Schiavina R, Martinelli A, et al. Diagnostic role of circulating free plasma DNA detection in patients with localized prostate cancer. Am J Clin Pathol 2008;129:756-62.
13. Chun FK, Muller I, Lange I, Friedrich MG, Erbersdobler A, Karakiewicz PI, et al. Circulating tumour-associated plasma DNA represents an independent and informative predictor of prostate cancer. Br J Urol Int 2006;98:544-8.

14. Xia P, Radpour R, Zachariah R, Fan AX, Kohler C, Hahn S, et al. Simultaneous quantitative assessment of circulating cellfree mitochondrial and nuclear DNA by multiplex real-time PCR. Genet Mol Biol [online] 2009;32:20-4.

15. Guo W, Jiang L, Bhasin S, Khan SM, Swerdlow RH. DNA extraction procedures meaningfully influence qPCR-based mtDNA copy number determination. Mitochondrion 2009;9:261-5.

16. Troeger C, Zhong XY, Burgemeister R, Minderer S, Tercanli S, Holzgreve W, et al. Approximately half of the erythroblasts in maternal blood are of fetal origin. Mol Hum Reprod 1999;5: 1162-5.

17. Szpechcinski A, Struniawska R, Zaleska J, Chabowski M, Orlowski T, Roszkowski K, et al. Evaluation of fluorescencebased methods for total vs. amplifiable DNA quantification in plasma of lung cancer patients. J Physiol Pharmacol 2008;59(Suppl 6):675-81.

18. Zhong XY, Hahn S, Kiefer V, Holzgreve W. Is the quantity of circulatory cell-free DNA in human plasma and serum samples associated with gender, age and frequency of blood donations? Ann Hematol 2007;86:139-43.

19. Aplenc R, Orudjev E, Swoyer J, Manke B, Rebbeck T. Differential bone marrow aspirate DNA yields from commercial extraction kits. Leukemia 2002;16:1865-6.

20. Carter MJ, Milton ID. An inexpensive and simple method for DNA purifications on silica particles. Nucleic Acids Res 1993;21:1044.

21. Liu KJ, Brock MV, Shih Ie M, Wang TH. Decoding circulating nucleic acids in human serum using microfluidic single molecule spectroscopy. J Am Chem Soc 2010;132:5793-8.

22. Wang M, Block TM, Steel L, Brenner DE, Su YH. Preferential isolation of fragmented DNA enhances the detection of circulating mutated k-ras DNA. Clin Chem 2004;50:211-3. 